\title{
Sequential share bargaining
}

Citation for published version (APA):

Herings, P. J. J., \& Predtetchinski, A. (2007). Sequential share bargaining. METEOR, Maastricht University School of Business and Economics. METEOR Research Memorandum No. 005 https://doi.org/10.26481/umamet.2007005

Document status and date:

Published: 01/01/2007

DOI:

10.26481/umamet.2007005

Document Version:

Publisher's PDF, also known as Version of record

\section{Please check the document version of this publication:}

- A submitted manuscript is the version of the article upon submission and before peer-review. There can be important differences between the submitted version and the official published version of record.

People interested in the research are advised to contact the author for the final version of the publication, or visit the DOI to the publisher's website.

- The final author version and the galley proof are versions of the publication after peer review.

- The final published version features the final layout of the paper including the volume, issue and page numbers.

Link to publication

\footnotetext{
General rights rights.

- You may freely distribute the URL identifying the publication in the public portal. please follow below link for the End User Agreement:

www.umlib.nl/taverne-license

Take down policy

If you believe that this document breaches copyright please contact us at:

repository@maastrichtuniversity.nl

providing details and we will investigate your claim.
}

Copyright and moral rights for the publications made accessible in the public portal are retained by the authors and/or other copyright owners and it is a condition of accessing publications that users recognise and abide by the legal requirements associated with these

- Users may download and print one copy of any publication from the public portal for the purpose of private study or research.

- You may not further distribute the material or use it for any profit-making activity or commercial gain

If the publication is distributed under the terms of Article $25 \mathrm{fa}$ of the Dutch Copyright Act, indicated by the "Taverne" license above, 
P. Jean-Jacques Herings, Arkadi Predtetchinski

Sequential Share Bargaining

RM/07/005

JEL code : C78

\section{METE@R}

Maastricht research school of Economics of TEchnology and ORganizations

Universiteit Maastricht

Faculty of Economics and Business Administration

P.O. Box 616

NL - 6200 MD Maastricht

phone : ++31433883830

fax $\quad$ : ++31433884873 



\title{
Sequential Share Bargaining*
}

\author{
P. Jean-Jacques Herings ${ }^{\dagger} \quad$ Arkadi Predtetchinski ${ }^{\ddagger}$ \\ February 13, 2007
}

\begin{abstract}
This paper presents a new extension of the Rubinstein-Ståhl bargaining model to the case with $n$ players, called sequential share bargaining. The bargaining protocol is natural and has as its main feature that the players' shares in the cake are determined sequentially. The bargaining protocol requires unanimous agreement for proposals to be implemented. Unlike all existing bargaining protocols with unanimous agreement, the resulting game has unique subgame perfect equilibrium utilities for any value of the discount factor. In equilibrium, agreement is reached immediately. The results are therefore qualitatively the same as in the two player case. The result builds on an analysis of so-called one-dimensional bargaining problems. We show that also one-dimensional bargaining problems have unique subgame perfect equilibrium utilities for any value of the discount factor, and that also in one-dimensional bargaining problems agreement is reached immediately.
\end{abstract}

Keywords: Noncooperative bargaining, dynamic games, subgame perfect equilibrium, unanimous agreement.

\section{JEL code: C78.}

${ }^{*}$ The authors would like to thank the Netherlands Organisation for Scientific Research (NWO) for financial support.

†P.J.J. Herings, Department of Economics, Maastricht University, P.O. Box 616, 6200 MD Maastricht, The Netherlands, e-mail: P.Herings@algec.unimaas.nl.

$\ddagger$ A. Predtetchinski, Department of Economics, Maastricht University, P.O. Box 616, 6200 MD Maastricht, The Netherlands, e-mail: A.Predtetchinski@algec.unimaas.nl. 


\section{Introduction}

In many socioeconomic problems, parties can create a surplus by collaborating. Bargaining problems study the distribution of the surplus over the parties involved. In the strategic theory of bargaining, a detailed process of negotations concerning the surplus is described, which is then analyzed by the tools of game theory. A commonly studied negotiation process is the one of alternating offers bargaining, first studied by Ståhl (1972) under the assumption of an exogenous deadline, next extended by Rubinstein (1982) to the case of an infinite horizon.

In the Rubinstein-Ståhl bargaining model, two players have to reach an agreement on the partition of a cake of a given size. Players make in turn a proposal as to how to divide the cake. After an offer by a player, the other player decides whether to accept it, or to reject it and continue the bargaining process by making an offer himself. After acceptance, a proposal is implemented. The game has a unique subgame perfect equilibrium under weak assumptions. In the unique subgame perfect equilibrium, the proposal of the first proposer is immediately accepted by his opponent.

This analysis does not carry over to bargaining problems with $n$ players. As reported in Osborne and Rubinstein (1990), a first extension to the $n$-person case is due to Shaked and consists of an example involving three players. In this example, player 1 starts by making a public proposal about splitting the cake to the other two players. A proposal consists of specifying a share in the cake for each of the players. The other players must accept or reject this proposal sequentially. If all agree, the proposal is implemented, otherwise it is rejected, one period of time elapses, and the next player makes a new proposal. Bargaining continues in this way. Herrero (1985) and Haller (1986) show that there is no unique subgame perfect equilibrium for the $n$-person case if the discount factor is sufficiently high. In particular, any feasible agreement is supported by a subgame perfect equilibrium, and equilibria with arbitrarily long delay exist.

Alternative extensions of the Rubinstein-Ståhl bargaining model are given by Jun (1987), Chae and Yang (1988), Yang (1992), Chae and Yang (1994), Krishna and Serrano (1996), Huang (2002), and Suh and Wen (2006). These authors consider games with partial agreement, also referred to as exit games. In an exit game, players need not agree unanimously to a proposal. In case of partial agreement, those players who have accepted the proposal may exit the game with the shares awarded by the proposer. These papers reproduce the basic results of the 2 -player case for the $n$-player case. Under weak assumptions, a unique subgame perfect equilibrium exists, and in this equilibrium agreement is reached without delay.

This paper studies $n$-person bargaining problems where unanimous agreement of all players is needed before an agreement can be implemented. This feature is common to many 
real-life bargaining situations, where it is not allowed for players to leave the bargaining table with only partial agreements of others.

Our bargaining protocol is as follows. An agenda specifies the order in which players' shares must be determined. The share of the player who is on top of the agenda, say player 1, is being negotiated first. Once all players agree on the share of player 1, this player exits the game and the remaining players proceed to negotiate over the share of the player who is next on the agenda, say player 2. Once all players agree on player 2's share, the remaining players negotiate over the share of the next player on the agenda, and so on, and so forth. We refer to this bargaining procedure as sequential share bargaining.

Suppose player $i$ 's share is being negotiated in period $t$. The players whose share has not been determined yet are called the active players. Nature selects one of the active players to make a proposal. This player proposes the share of the cake to be allocated to player $i$. The remaining players react, sequentially, to the proposal. If the proposal is unanimously agreed upon, player $i$ obtains his share and exits the game. The remaining players then proceed to negotiate over the share of the next player on the agenda. Without unanimous agreement, period $t$ ends and time moves to period $t+1$. The utility of a player who obtains a share $x$ of the cake in period $t$ is $\delta^{t} x$, where $\delta$ is the common discount factor.

In determining the share of player $i$, it is natural to think about player $i$ as being involved in a bargaining situation between two coalitions. One coalition consists of player $i$ himself, the other coalition of all opponents of player $i$. All players in the latter coalition share a preference for making the share awarded to player $i$ as small as possible.

To solve the game resulting from the sequential share bargaining procedure, we first study so-called one-dimensional bargaining problems. A one-dimensional bargaining problem consists of two rival coalitions that bargain over the choice of $x$ in an interval $[0, X]$, where $X>0$ is the surplus that the rival coalitions have to share. The utility functions of players in $S$ are identical and monotonically increasing in $x$. The utility functions of players in $T$ are identical too, but monotonically decreasing in $x$. One-dimensional bargaining problems are also studied in Banks and Duggan (2000) and Cho and Duggan (2003).

Although we need the one-dimensional bargaining problem as a building block to obtain results for sequential share bargaining, one-dimensional bargaining problems are worth studying in their own right. Many real-life bargaining situations can be approximated by the case where the bargaining space is one-dimensional, the players involved can be partitioned in two groups, with preferences within the group identical, and between groups diametrically opposed. Examples include the division of a fixed budget over two possible goals, the location of a public facility on a line, and negotiations between two firms (where a firm is viewed upon as a collection of agents with identical preferences) or between a firm and an individual about the price of a product or service. 
We show that one-dimensional bargaining leads to unique subgame perfect equilibrium utilities. A subgame perfect equilibrium is characterized by the absence of delay. We provide a linear system of characteristic equations that makes the computation of the equilibrium strategy profile an easy task. Cho and Duggan (2003) consider a one-dimensional bargaining model with quadratic utilities and decision making by a coalition in a set of decisive coalitions. For the Cho and Duggan specification of the model, the concept of subgame perfect equilibrium in stationary strategies is needed to obtain uniqueness of equilibrium. Surprisingly, in our model subgame perfection suffices to obtain unique predictions.

From our results on one-dimensional bargaining problems, we derive by means of the following induction argument that the subgame perfect equilibrium utilities in sequential share bargaining are uniquely determined. Clearly, 1-person sequential share bargaining problems have unique subgame perfect equilibrium utilities. The unique equilibrium utilities of an $n$-person sequential share bargaining situation can be substituted in all subgames of an $(n+1)$-person sequential share bargaining problem where the share of the first person on the agenda has been decided upon. The resulting reduced sequential share bargaining game belongs to the class of one-dimensional bargaining problems, having unique subgame perfect equilibrium utilities. Moreover, the equilibria in sequential share bargaining games are characterized by absence of delay.

Section 2 introduces the one-dimensional bargaining problem and Section 3 its characteristic equations. In Section 4 it is shown that the one-dimensional bargaining problem leads to unique subgame perfect equilibrium utilities. Section 5 defines the game underlying the sequential share bargaining procedure. Building on the result of Section 4, it is shown in Section 6 that sequential share bargaining leads to unique subgame perfect equilibrium utilities. Section 7 concludes.

\section{One-dimensional Bargaining}

This section studies one-dimensional bargaining with unanimous agreement. In a onedimensional bargaining game $\hat{\Gamma}$, a finite set of players $N$ has to agree on the choice of $x$ in a non-degenerate interval $[0, X]$. The $n$ players in $N$ are partitioned in the non-empty coalitions $S$ and $T$. All players in $S$ have identical preferences that are monotonically increasing in $x$. All players in $T$ have identical preferences that are monotonically decreasing in $x$.

The game $\hat{\Gamma}$ is a dynamic game of perfect information in discrete time. At each time period $t=0,1, \ldots$ nature selects a proposer from the set $N$. The chosen player makes a proposal, i.e. a choice for $x$ in the interval $[0, X]$. We denote a proposal by player $i \in N$ by $x_{i}$. The remaining players respond, sequentially, to the proposal. We assume that first all 
players belonging to the proposer's coalition respond, followed by all players in the other coalition. Either a proposal is unanimously accepted, it is implemented, and the game ends. Or some player rejects the proposal, period $t+1$ begins, and nature selects a new proposer. An outcome of the game is either a perpetual disagreement or a pair $(t, x)$, i.e. $x$ is agreed upon in period $t$.

To select a proposer, nature chooses according to a Markov process with state space $N$. The transition probabilities of the process are given by the transition matrix $\pi$. Thus $\pi_{i j}$ is the probability of a transition from state $i$ to state $j$, i.e. if the last proposer has been player $i$, then with probability $\pi_{i j}$ the next proposer is player $j$. The first proposer is chosen according to a probability distribution $\pi^{0}$ on $N$.

The players in coalition $S$ have identical preferences over outcomes. The utility of a player $i \in S$ who receives outcome $x$ in period $t$ is $u_{i}(t, x)=\delta^{t} x$, with the utility of perpetual disagreement being 0 . Similarly, the players in $T$ have identical preferences. The utility of a player $i \in T$ who receives outcome $x$ in period $t$ is $u_{i}(t, x)=\delta^{t}(X-x)$, with the utility of perpetual disagreement being 0 . Here, $\delta \in(0,1)$ is the common discount factor. A one-dimension bargaining game is $\hat{\Gamma}=\left\{S, T, X, \delta,\left(u_{i}\right)_{i \in S \cup T}, \pi^{0}, \pi\right\}$.

When $S$ and $T$ are both singletons, the game $\hat{\Gamma}$ is a two-player game and contains Rubinstein (1982) as a special case. Merlo and Wilson (1995) have generalized the Rubinstein set-up substantially and allow for a proposer selected by a Markov process. But since they consider bargaining problems where the dimension of the bargaining space equals the number of players minus one, one-dimensional bargaining with more than two players is not covered by their analysis.

Since members of a given coalition have identical preferences, it is tempting to assume that they should adopt the same strategy. Then each coalition can be seen a single player and the game $\hat{\Gamma}$ is in essence a two-player game. This reasoning is not correct, even if one restricts attention to stationary strategies. The reason is that though preferences are identical within a coalition, the transition probabilities $\pi$ depend on the identity of the proposer, implying that different players of the same coalition have different positions in the bargaining game. As a consequence, different members of a given coalition may find it optimal to make different proposals.

\section{The Characteristic Equations for One-dimensional Bargaining}

In this section we derive a subgame perfect equilibrium of the game $\hat{\Gamma}$ from the solution to a linear system of characteristic equations.

For a player $i$ in $N$ and a coalition $C$, i.e. a member of $\{S, T\}$, the variable $z_{C}^{i}$ denotes 
the continuation utility of a member of coalition $C$ after the rejection of a proposal made by player $i$. All members of a given coalition have the same preferences, so receive the same utility in any outcome of the game. The characteristic equations of $\hat{\Gamma}$ describe a particular subgame perfect equilibrium in terms of the variables $z_{C}^{i}$. The characteristic equations are as follows:

$$
\begin{aligned}
z_{T}^{i}=\pi_{i T} X+\delta \sum_{j \in S} \pi_{i j} z_{T}^{j}-\delta \sum_{j \in T} \pi_{i j} z_{S}^{j}, \quad i \in N \\
z_{S}^{i}=\pi_{i S} X-\delta \sum_{j \in S} \pi_{i j} z_{T}^{j}+\delta \sum_{j \in T} \pi_{i j} z_{S}^{j}, \quad i \in N,
\end{aligned}
$$

where $\pi_{i C}=\sum_{j \in C} \pi_{i j}$. This is a system of $2 n$ equations and $2 n$ unknowns.

The idea behind system (1)-(2) is that a proposal of any member of coalition $C$ leaves any member of the rival coalition $N \backslash C$ indifferent between accepting and rejecting the proposal. Thus, a player $j \in S$ makes a proposal $X-\delta z_{T}^{j}$. Such a proposal makes all members of coalition $T$ indifferent between acceptance and rejection, since either action results in utility $\delta z_{T}^{j}$. Similarly, a player $j \in T$ makes a proposal $\delta z_{S}^{j}$. Such a proposal makes each member of $S$ indifferent between acceptance and rejection, since either action results in utility $\delta z_{S}^{j}$.

Now suppose that a proposal of player $i$ has been rejected. Then the continuation utility of any member of coalition $S$ is

$$
\sum_{j \in S} \pi_{i j}\left(X-\delta z_{T}^{j}\right)+\sum_{j \in T} \pi_{i j} \delta z_{S}^{j} .
$$

Setting this expression equal to $z_{S}^{i}$ gives equation (2). In a similar way, we find that the continuation utility of any member of $T$ equals

$$
\sum_{j \in S} \pi_{i j} \delta z_{T}^{j}+\sum_{j \in T} \pi_{i j}\left(X-\delta z_{S}^{j}\right)
$$

Setting this expression equal to $z_{T}^{i}$ gives equation (1).

Theorem 3.1 The system of characteristic equations (1)-(2) has a unique solution.

Proof. Adding up equations (1) and (2) for fixed $i$ we obtain the equation $z_{T}^{i}+z_{S}^{i}=X$. We can therefore express each $z_{T}^{j}$ as $X-z_{S}^{j}$ and substitute this into equations (2). This yields

$$
z_{S}^{i}=(1-\delta) \pi_{i S} X+\delta \sum_{j \in N} \pi_{i j} z_{S}^{j}
$$

It is sufficient to show that system (3) has a unique solution. System (3) is a system of $n$ equations and $n$ unknowns that can be rewritten in vector-matrix notation as $z_{S}=y+\delta \pi z_{S}$. 
Because $\pi$ is a row-stochastic matrix, the spectral radius of $\pi$ is at most 1 . It follows that the matrix $I-\delta \pi$ is invertible, where $I$ is the identity matrix. The result follows.

We define a profile of strategies $\hat{\sigma}=\left(\hat{\sigma}^{i}\right)_{i \in N}$ that will be verified to constitute a subgame perfect equilibrium of $\hat{\Gamma}$. Let the $2 n$-dimensional vector $z$ be a solution to the system of characteristic equations (1)-(2). The behavioral strategy of a player $i \in S$ is defined as follows. Whenever player $i$ is selected to make a proposal, he proposes $x_{i}=X-\delta z_{T}^{i}$, and whenever player $i$ is selected to respond to a proposal $x_{j}$ of player $j \in N$, he accepts if and only if $x_{j} \geq \delta z_{S}^{j}$. The behavioral strategy of a player $i \in T$ is defined as follows. Whenever player $i$ is selected to make a proposal, he proposes $x_{i}=\delta z_{S}^{i}$, and whenever player $i$ is selected to respond to a proposal $x_{j}$ of player $j \in N$, he accepts if and only if $x_{j} \leq X-\delta z_{T}^{j}$.

When players play according to strategy profile $\hat{\sigma}$, bargaining proceeds as follows. If player $i \in S$ is selected to make a proposal, he proposes $x_{i}=X-\delta z_{T}^{i}$. Next the players in $S$ respond. They accept if and only if $x_{i} \geq \delta z_{S}^{i}$. This inequality holds strictly, since $z_{S}^{i}+z_{T}^{i}=X$ and $\delta<1$, so as a consequence all players $i \in S$ accept. Next the players in $T$ respond. They accept if and only if $x_{i} \leq X-\delta z_{T}^{i}$, an inequality that holds with equality, so all players in $T$ accept.

If a player $i \in T$ is selected to make a proposal, bargaining proceeds in basically the same way. His proposal $x_{i}$ equals $\delta z_{S}^{i}$, which is first accepted by all responders in $T$ and next accepted by all responders in $S$.

Theorem 3.2 The strategy profile $\hat{\sigma}$ is a subgame perfect equilibrium of the game $\hat{\Gamma}$.

Proof. Consider a subgame $\hat{\Gamma}(h)$ of $\hat{\Gamma}$ that starts at node $h$ of $\hat{\Gamma}$. Suppose a player $i \in N$ has a profitable deviation, which increases the subgame utility by $\varepsilon>0$. Since the utility player $i$ can get from a node that is $t$ periods later than the initial node of the subgame is bounded by $\delta^{t}$, player $i$ has a profitable deviation $\bar{\sigma}^{i}$ that deviates from $\hat{\sigma}^{i}$ only at nodes corresponding to the first $T$ periods, where $T$ equals $\ln (\varepsilon) / \ln (\delta)$.

Consider a node $h^{\prime}$ where player $i$, when playing according to $\bar{\sigma}^{i}$, deviates from $\hat{\sigma}^{i}$, and which is not succeeded by another node where $i$ deviates from $\hat{\sigma}^{i}$. Consider the subsubgame $\hat{\Gamma}\left(h^{\prime}\right)$ starting at this node. Then either $\bar{\sigma}^{i}$ induces a profitable deviation in the subsubgame, or the strategy $\tilde{\sigma}^{i}$ that is equal to $\bar{\sigma}^{i}$, except at $h^{\prime}$, where $\tilde{\sigma}^{i}\left(h^{\prime}\right)=\hat{\sigma}^{i}\left(h^{\prime}\right)$, is a profitable deviation from $\hat{\sigma}^{i}$ in subgame $\Gamma(h)$. Iterating this argument, we can show that there is a subgame $\hat{\Gamma}\left(h^{0}\right)$ of $\hat{\Gamma}$ such that player $i$ acts at node $h^{0}$ and player $i$ has a profitable deviation which only deviates from $\hat{\sigma}$ at $h^{0}$.

Consider the subgame $\hat{\Gamma}\left(h^{0}\right)$. We complete the proof by showing that a one-shot deviation from $\hat{\sigma}^{i}$ cannot by profitable. To simplify the notation, we divide utility by $\delta^{t}$, where $t$ is the period corresponding to the subgame's first node $h^{0}$. 
Suppose player $i \in S$ is a proposer in the first node of $\hat{\Gamma}\left(h^{0}\right)$. The use of strategy $\hat{\sigma}^{i}$ leads to a proposal $x_{i}=X-\delta z_{T}^{i}$, which is unanimously accepted by all responders, and leads to utility $X-\delta z_{T}^{i}$ for player $i$.

Proposing $x>x_{i}$ leads to rejection by a player in $T$. Whenever players $j \in S$ are next selected as a proposer, they propose $X-\delta z_{T}^{j}$, and players $j \in T$ propose $\delta z_{S}^{j}$. The utility of player $i$ is therefore equal to

$$
\delta\left(\sum_{j \in S} \pi_{i j}\left(X-\delta z_{T}^{j}\right)+\sum_{j \in T} \pi_{i j} \delta z_{S}^{j}\right) .
$$

Since $z$ is a solution to the characteristic equations, this expression equals $\delta z_{S}^{i}$. From $\delta z_{S}^{i}+\delta z_{T}^{i}=\delta X$, it follows that $\delta z_{S}^{i}<X-\delta z_{T}^{i}$, and player $i$ looses utility by proposing $x>x_{i}$.

Now consider a proposal $x$ by $i$ satisfying $x<x_{i}$. If this proposal is accepted, it leads to utility for $i$ less than $X-\delta z_{T}^{i}$. If it is rejected, then by the same argument as before, it will lead to utility $\delta z_{S}^{i}$, which is less than $X-\delta z_{T}^{i}$.

Suppose player $i \in T$ is a proposer in the first node of $\hat{\Gamma}\left(h^{0}\right)$. Then a fully analogous argument shows that he does not have a profitable one-shot deviation.

Suppose player $i \in S$ is a responder in the first node of $\hat{\Gamma}\left(h^{0}\right)$ and responds to a proposal $x \in[0, X]$ by a player $j$. If $i$ is asked to respond to this proposal, then according to $\hat{\sigma}^{i}$ acceptance takes place if $x \geq \delta z_{S}^{j}$, and results in utility equal to $x$ if all players responding after player $i$ accept $x$ or to $\delta z_{S}^{j}$ otherwise. A deviation to rejection leads to utility $\delta z_{S}^{j}$, and is therefore not profitable. If $x<\delta z_{S}^{j}$, then player $i$ rejects the proposal when playing according to $\hat{\sigma}^{i}$, and obtains utility $\delta z_{S}^{j}$. A deviation from rejection to acceptance results in utility equal to $x$ if all players responding after player $i$ accept $x$ or to $\delta z_{S}^{j}$ otherwise, and is therefore not profitable.

Suppose player $i \in T$ is a responder in the first node of the subgame and responds to a proposal $x \in[0, X]$ by a player $j$. Then a fully analogous argument shows that he does not have a profitable one-shot deviation.

Example 3.1. Suppose the coalition $S$ is a singleton consisting of player $i_{1}, T=\left\{i_{2}, \ldots, i_{n}\right\}$, and the identity of the proposer cycles within the player set: $i_{1}, i_{2}, i_{3}, \ldots, i_{n}, i_{1}$. The system of characteristic equations (1)-(2) yields

$$
\begin{aligned}
z_{T}^{i_{1}} & =X-\delta z_{S}^{i_{2}} \\
z_{S}^{i_{j}} & =\delta z_{S}^{i_{j+1}}, j=2, \ldots, n-1 \\
z_{S}^{i_{n}} & =X-\delta z_{T}^{i_{1}}
\end{aligned}
$$


Solving it, we find

$$
z_{T}^{i_{1}}=\frac{1-\delta^{n-1}}{1-\delta^{n}} X,
$$

so the equilibrium proposal of player $i_{1}$ equals

$$
x_{i_{1}}=X-\delta z_{T}^{i_{1}}=\frac{1-\delta}{1-\delta^{n}} X .
$$

This proposal will be accepted by all players in equilibrium. The equilibrium utility of player $i_{1}$ is

$$
\frac{1-\delta}{1-\delta^{n}} X
$$

and the equilibrium utility of players in $T$ is

$$
\frac{\delta-\delta^{n}}{1-\delta^{n}} X .
$$

Since $1-\delta$ does not necessarily exceed $\delta-\delta^{n}$, the equilibrium utility of the first mover, player $i_{1}$, may be lower than that of the players in $T$.

In subgames (that will not be reached in equilibrium) where a player $i_{k}, k=2, \ldots, n$, has to make a proposal, it is equal to

$$
x_{i_{k}}=\delta^{n-k+1} \frac{1-\delta}{1-\delta^{n}} X,
$$

which equals $\delta^{n-k+1}$ times the proposal of player $i_{1}$.

In a subgame where player $i_{2}$ makes a proposal, he may propose a low value of $x$, since a long time will elapse before a player belonging to the opposing coalition can make a proposal. In a subgame where player $i_{n}$ makes a proposal, he knows that player $i_{1}$ will be the next proposer, so he proposes a relatively high value of $x$. Observe that we obtain the Rubinstein (1982) result for the case where $n=2$.

If, for fixed $\delta$, the number of players $n$ goes to infinity, then the equilibrium proposal of player $i_{1}$ converges to $(1-\delta) X$, the proposal of player $i_{2}$ to 0 , and the proposal of player $i_{n}$ to $\delta(1-\delta) X$. If, for a fixed number of players, $\delta$ converges to 1 , then the equilibrium proposals of all players converge to $X / n$.

Example 3.2. Suppose the coalition $S$ consists of the first $k$ agents, coalition $T$ equals $N \backslash\{1, \ldots, k\}$, and players are selected randomly to be the proposer, $\pi_{i}^{0}=1 / n$ for all $i \in N$, and $\pi_{i j}=1 / n$ for all $i, j \in N$. For $i \in N$, the system of characteristic equations (1)-(2) is then equal to

$$
\begin{aligned}
& z_{T}^{i}=\frac{n-k}{n} X+\delta \sum_{j \in S} \frac{1}{n} z_{T}^{j}-\delta \sum_{j \in T} \frac{1}{n} z_{S}^{j}, \\
& z_{S}^{i}=\frac{k}{n} X-\delta \sum_{j \in S} \frac{1}{n} z_{T}^{j}+\delta \sum_{j \in T} \frac{1}{n} z_{S}^{j} .
\end{aligned}
$$


Solving it, we find

$$
\begin{aligned}
z_{S}^{i} & =\frac{k}{n} X, \\
z_{T}^{i} & =\frac{n-k}{n} X .
\end{aligned}
$$

The equilibrium proposal of a player $i=1, \ldots, k$ equals

$$
x_{i}=X-\delta z_{T}^{i}=\frac{\delta k+(1-\delta) n}{n} X=\delta \frac{k}{n} X+(1-\delta) X .
$$

This proposal will be accepted by all players in equilibrium. The equilibrium proposal of a player $i=k+1, \ldots, n$ is given by

$$
x_{i}=\delta \frac{k}{n} X .
$$

The larger the ratio $k / n$, the higher the fraction of players belonging to coalition $S$, and the higher the proposed value of $x$.

The expected utility of a player $i \in S$ is given by

$$
\frac{k}{n}\left(\delta \frac{k}{n} X+(1-\delta) X\right)+\frac{n-k}{n} \delta \frac{k}{n} X=\frac{k}{n} X .
$$

Also the expected proposal is equal to $(k / n) X$. The expected utility of a player $i \in T$ equals $((n-k) / n) X$. In this example, expected utilities are equal to expected continuation utilities.

If, for fixed $\delta$ and a fixed size of coalition $S$, the number of players in $T$ goes to infinity, then the equilibrium proposal of players in $S$ converges to $(1-\delta) X$, and the proposal of players in $T$ converges to 0 . If, for fixed $\delta$ and a fixed size of coalition $T$, the number of players in $S$ goes to infinity, then the equilibrium proposal of players in $S$ converges to $X$, and the proposal of players in $T$ converges to $\delta X$. If, for a fixed number of players, $\delta$ converges to 1 , then the equilibrium proposals of all players converge to $(k / n) X$.

\section{Uniqueness of Equilibrium in One-dimensional Bar- gaining Games}

In this section we show that subgame perfect equilibrium utilities are unique, and therefore correspond to the ones following from $\hat{\sigma}$.

For $i \in N$, let $\hat{\Gamma}^{i}$ denote the class of subgames of the game $\hat{\Gamma}$ starting with player $i$ in the role of proposer. Since all subgames in $\hat{\Gamma}^{i}$ are strategically equivalent, the set of subgame perfect equilibrium strategies of $\hat{\Gamma}^{i}$ is well-defined. The utilities of two games 
in the class $\hat{\Gamma}^{i}$ differ from each other only by a power of $\delta$. The set of subgame perfect equilibrium utilities in a game in $\hat{\Gamma}^{i}$ discounted by $1 / \delta^{t}$, where $t$ denotes the starting period of the game, is the same for all games in $\hat{\Gamma}^{i}$.

For $i \in N$, and $C \in\{S, T\}$, let $\underline{u}_{C}^{i}\left(\bar{u}_{C}^{i}\right)$ be the infimum (supremum) of the discounted utilities to coalition $C$ over all subgame perfect equilibria of $\hat{\Gamma}^{i}$. Let $\underline{z}_{C}^{i}\left(\bar{z}_{C}^{i}\right)$ be the infinum (supremum) of the continuation utilities to coalition $C$ following the rejection of a proposal by player $i$ over all subgame perfect equilibria of the game $\hat{\Gamma}^{i}$.

The following result asserts that, for $i \in N$, for $C \in\{S, T\}, \underline{u}_{C}^{i}=\bar{u}_{C}^{i}$, and $\underline{z}_{C}^{i}=\bar{z}_{C}^{i}$. This result implies that subgame perfect equilibrium utilities are unique.

Theorem 4.1 For any $\hat{\Gamma}$, for $i \in N$ and $C \in\{S, T\}, \underline{u}_{C}^{i}=\bar{u}_{C}^{i}$ and $\underline{z}_{C}^{i}=\bar{z}_{C}^{i}$. In any subgame perfect equilibrium, agreement is reached without delay.

Proof. Since rejection of a proposal by player $i$ leads to a subgame in $\hat{\Gamma}^{j}$ with probability $\pi_{i j}$, it holds that $\underline{z}_{C}^{i} \geq \sum_{j \in N} \pi_{i j} \underline{u}_{C}^{j}$. Similarly, it can be derived that $\bar{z}_{C}^{i} \leq \sum_{j \in N} \pi_{i j} \bar{u}_{C}^{j}$. Let $\Delta$ be the maximum of the differences $\bar{u}_{C}^{i}-\underline{u}_{C}^{i}$ over all $i$ and $C$. Then $\bar{z}_{C}^{i}-\underline{z}_{C}^{i} \leq \Delta$ for all $i$ and $C$. We show that $\Delta=0$.

First we establish the following inequalities. For $C \in\{S, T\}$, for all $i \in C$,

$$
\begin{aligned}
& \delta \underline{z}_{N \backslash C}^{i} \leq \underline{u}_{N \backslash C}^{i} \leq \bar{u}_{N \backslash C}^{i} \leq \delta \bar{z}_{N \backslash C}^{i}, \\
& X-\delta \bar{z}_{N \backslash C}^{i} \leq \underline{u}_{C}^{i} \leq \bar{u}_{C}^{i} \leq X-\delta \underline{z}_{N \backslash C}^{i} .
\end{aligned}
$$

We start with $C=T$. The inequality $\delta \underline{z}_{S}^{i} \leq \underline{u}_{S}^{i}$ follows from the fact that when a player of coalition $S$ rejects a proposal of player $i \in T$, he obtains a utility of at least $\underline{z}_{S}^{i}$ in the next period. His subgame perfect equilibrium utility can therefore not be less than $\delta \underline{z}_{S}^{i}$.

Let $\left(v_{S}^{i}, v_{T}^{i}\right)$ be subgame perfect equilibrium utilities. Then $v_{T}^{i} \leq X-v_{S}^{i}$, where the inequality comes from the fact that there might be delay before an agreement is reached, so utilities may sum to less than $X$. As a consequence, $\bar{u}_{T}^{i} \leq X-\underline{u}_{S}^{i}$, which yields the inequality $\bar{u}_{T}^{i} \leq X-\delta \underline{z}_{S}^{i}$.

Now suppose player $i \in T$ makes a proposal $x>\delta \bar{z}_{S}^{i}$. We will argue that in a subgame perfect equilibrium this leads to utilities of at least $X-x$ to $i$, and therefore to players in $T$. First the players in $T$ respond in the order $i_{1}, \ldots, i_{\ell}$, next the players in $S$ in the sequence $j_{1}, \ldots, j_{k}$. If player $j_{k}$ is given the option to respond, it means that all other players have accepted the proposal. If player $j_{k}$ accepts, his utility is $x$, otherwise it is at most $\delta \bar{z}_{S}^{i}$. Player $j_{k}$ will accept therefore. By a backwards induction argument it follows that all players $j_{1}, \ldots, j_{k}$ will accept the proposal. Consider next player $i_{\ell}$. Acceptance by player $i_{\ell}$ leads to utilities $X-x$, rejection will therefore only occur if it leads to utilities at least equal to $X-x$, meaning that the utility to any player in $T$ is at least $X-x$. By a backwards induction argument it follows that player $i_{1}$, the first to respond, can ensure 
a utility of at least $X-x$ by accepting $x$. Thus, player $i$ can guarantee himself a utility of at least $X-x$ for any $x>\delta \bar{z}_{S}^{i}$ by proposing $x$. This shows that $X-\delta \bar{z}_{S}^{i} \leq \underline{u}_{T}^{i}$. The inequality $\bar{u}_{S}^{i} \leq \delta \bar{z}_{S}^{i}$ follows. The case $C=S$ is similar.

The above inequalities imply that for all $C \in\{S, T\}$, for $i \in C$,

$$
\bar{u}_{N \backslash C}^{i}-\underline{u}_{N \backslash C}^{i} \leq \delta\left(\bar{z}_{N \backslash C}^{i}-\underline{z}_{N \backslash C}^{i}\right) \text { and } \bar{u}_{C}^{i}-\underline{u}_{C}^{i} \leq \delta\left(\bar{z}_{N \backslash C}^{i}-\underline{z}_{N \backslash C}^{i}\right) .
$$

Since $\Delta$ is defined as the maximum of the differences $\bar{u}_{C}^{i}-\underline{u}_{C}^{i}$ over $i$ and $C$, we can derive the inequality $\Delta \leq \delta \Delta$. Thus $\Delta=0$, as desired.

We have thus proved that for all $i \in N$, for all $C \in\{S, T\}, \underline{u}_{C}^{i}=\bar{u}_{C}^{i}$, and $\underline{z}_{C}^{i}=\bar{z}_{C}^{i}$.

Theorem 3.2 pins down the equilibrium utility levels. Since delay of agreements would lead to different utility levels, equilibrium agreement is reached without delay in any subgame perfect equilibrium.

In general, $\hat{\Gamma}$ may have more than one subgame perfect equilibrium, but the multiplicity is inessential in the following sense. Assume for instance that coalition $S$ consists of two players, $i_{1}$ and $i_{2}$, and suppose that the players respond to proposals in this order. If a player $j$ in $T$ makes a proposal smaller than $\delta z_{S}^{j}$, the players in $S$ will reject this proposal in any subgame perfect equilibrium. It is completely irrelevant, however, whether this proposal will be rejected by $i_{1}$, or whether $i_{1}$ accepts this proposal and has it rejected by $i_{2}$. In fact, even a member of $T$ different from $j$ may reject the, from his perspective, very favorable proposal, anticipating that some player in $S$ will reject it anyway. What matters is not the responses by individual players, but how the coalition $S \cup T \backslash\{j\}$ reacts to proposals.

Let $H$ be the set of all decision nodes of the extensive form game $\hat{\Gamma}$. We denote the decision nodes where some player has to make a proposal by $H^{p}$, and the decision nodes immediately following nodes in $H^{p}$, so nodes where the first player responds, by $H^{r}$. Given a strategy profile $\sigma$, for $h \in H^{p}, \sigma^{p}(h)$ denotes the proposal made at decision node $h$, and for $h \in H^{r}, \sigma^{r}(h)=1$ if the proposal is accepted by all players, and $\sigma^{r}(h)=0$, otherwise.

Let $\sigma$ be a subgame perfect equilibrium strategy profile. It is not hard to show that $\sigma$ is essentially equivalent to $\hat{\sigma}$ in the following sense. For any $h \in H^{p}, \sigma^{p}(h)=\hat{\sigma}^{p}(h)$. For any $h \in H^{r}$ where the proposal under discussion is $x$ by player $j \in S$, if $x \neq \delta z_{S}^{j}$, then $\sigma^{r}(h)=\hat{\sigma}^{r}(h)$. For any $h \in H^{r}$ where the proposal under discussion is $x$ by player $j \in T$, if $x \neq X-\delta z_{T}^{j}$, then $\sigma^{r}(h)=\hat{\sigma}^{r}(h)$. Subgame perfect equilibrium proposals are therefore unique. Only in subgames where by mistake a player $j \in S$ proposes $x_{j}=\delta z_{S}^{j}$, so leaving players in his own coalition indifferent between accepting and rejecting, or in subgames where by mistake a player $j \in T$ proposes $x_{j}=X-\delta z_{T}^{j}$, so leaving players in his own coalition indifferent between accepting and rejecting, could there be a difference in response behavior at the coalition level. 
Let $\hat{\Gamma}(X)$ be a one-dimensional bargaining game with cake size $X$. Consider any subgame perfect equilibrium of $\hat{\Gamma}(X)$. The continuation utility of a member of coalition $C$ after rejecting a proposal by player $i$ is $z_{C}^{i}(X)$. The variable $u_{C}^{i}(X)$ denotes the expected utility of a member of coalition $C$ in a subgame starting with a proposal by player $i$. The variable $u_{C}^{*}(X)$ is the expected utility of a player $i \in C$ as evaluated at the beginning of the game. The next result specifies how these variables can be computed, and claims that all utilities are linear functions of $X$. The latter property is crucial to derive our results for sequential share bargaining games.

Theorem 4.2 Consider any subgame perfect equilibrium of $\hat{\Gamma}(X)$. Then $\left(z_{S}^{i}(X), z_{T}^{i}(X)\right)_{i \in N}$ are given by the solution to (1)-(2). Moreover, for $C \in\{S, T\}$, for $i \in N \backslash C$,

$$
\begin{aligned}
u_{C}^{i}(X) & =\delta z_{C}^{i}(X), \\
u_{N \backslash C}^{i}(X) & =X-\delta z_{C}^{i}(X),
\end{aligned}
$$

and

$$
u_{C}^{*}(X)=\sum_{j \in N} \pi_{j}^{0} u_{N \backslash C}^{j}(X) .
$$

All the functions above are linear in $X$.

Proof. The expressions above follow in a straightforward way from Theorems 3.2 and 4.1. It follows from the proof of Theorem 3.1 that the solution to system (3) is given by $z_{S}(X)=(1-\delta) X(I-\delta \pi)^{-1} \pi_{S}$, where $\pi_{S}=\left(\left(\pi_{i S}\right)_{i \in N}\right)^{\top}$. It follows that $z_{S}^{i}(X)$ is linear in $X$. Since $z_{T}^{i}(X)=X-z_{S}^{i}(X)$, it holds that $z_{T}^{i}(X)$ is linear in $X$. Linearity of $u_{S}^{i}(X)$, $u_{T}^{i}(X), u_{S}^{*}(X)$, and $u_{T}^{*}(X)$ is now immediate.

\section{Sequential Share Bargaining}

In this section we consider the problem of dividing a cake of size $\bar{X}$ among $\bar{n}$ players. Our approach is to chop up the problem into $n$ one-dimensional bargaining procedures that are implemented sequentially, one after another. First, the players decide upon the share of the cake to be allocated to the first player on the agenda, say player 1. Once player 1's share is unanimously agreed upon by all active players, including player 1 , he leaves the game. The remaining players then decide what share of the cake to allocate to the next player on the agenda, say player 2. Once this is unanimously agreed upon, player 2 exits, and the remaining players decide upon the share of player 3 , and so on. 
A sequential share bargaining game $\Gamma$ is specified by a set of players $\bar{N}$ with cardinality $\bar{n}$, a cake size $\bar{X}$, a discount factor $\delta$, utility functions $\left(u_{i}\right)_{i \in \bar{N}}$, an agenda $\rho, \bar{n}$ initial probability distributions, $\left(\pi^{01}, \ldots, \pi^{0 \bar{n}}\right)$, and $\bar{n}$ Markov transition matrices, $\left(\pi^{1}, \ldots, \pi^{\bar{n}}\right)$.

All players have the same utility function. The utility of player $i \in \bar{N}$ when receiving the share $x$ in time period $t$ is $u_{i}(t, x)=\delta^{t} x$. The utility of perpetual disagreement is 0 .

The agenda $\rho$ is a permutation, an injective function mapping $\{1, \ldots, \bar{n}\}$ into the set of players $\bar{N}$. The agenda $\rho$ specifies the sequence in which the players' shares are decided upon. The player whose share is decided upon first is $\rho(1)$. If his share is specified, the remaining players $N=\bar{N} \backslash\{\rho(1)\}$ decide upon player $\rho(2)$ 's share. For $k=1, \ldots, \bar{n}$, $\rho^{k}=\left\{i \in \bar{N} \mid \rho^{-1}(i) \geq k\right\}$ denotes the set of players, whose position on the agenda is $k$ or higher. After the shares of players $\rho(1), \ldots, \rho(k-1)$ have been decided upon, the players in $\rho^{k}$ discuss the share of player $\rho(k)$.

The way to decide upon the share of a given player is the bargaining procedure as detailed below. Let $N=\rho^{k}$ be the set of players that determines the share of player $\rho(k)$ in a cake of size $X \leq \bar{X}$ in a time period $t$. Nature chooses a proposer from the set $N$ according to the probability distribution $\pi^{0 k}$, where $\pi_{i}^{0 k}$ is the probability that player $i$ is selected as a proposer. The chosen player announces a number $x \in[0, X]$, the share of the cake to be allocated to player $\rho(k)$. The remaining players respond sequentially to the proposal. If $\rho(k)$ is not the proposer himself, he is the last player to respond. If the proposal is unanimously accepted, then player $\rho(k)$ exits the game with share $x$. The players $\rho^{k+1}=N \backslash\{\rho(k)\}$ continue bargaining, and determine the share of player $\rho(k+1)$ in a cake of size $X-x$ in time period $t$. If the proposal is rejected, period $t+1$ begins and nature chooses a new proposer in $N$. The moves of nature that select the proposer follow a Markov process with $N$ as state space. The matrix of transition probabilities is given by $\pi^{k}$, where $\pi_{i j}^{k}$ is the transition probability from state $i$ to state $j$. The bargaining process proceeds in this way until the share of all players has been decided upon.

Our procedure differs from the bargaining games with exit as discussed in the introduction in that no player is allowed to take any part of the cake unless all other players agree. In contrast, in a game with exit, a player can receive a share offered to this player by a proposer without the consent of other players.

For $k=1, \ldots, \bar{n}$, and $0 \leq X \leq \bar{X}$, we denote a subgame of $\Gamma$ where the set of players $N=\rho^{k}$ determines the share of player $\rho(k)$ and the size of the cake is $X$ by $\Gamma(N, X)$. 


\section{Uniqueness of Equilibrium in Sequential Share Bar- gaining Games}

In this section we prove that sequential share bargaining games have unique subgame perfect equilibrium utilities. The gist of the argument is as follows. All sequential share bargaining games $\Gamma\left(\rho^{\bar{n}}, X\right)=\Gamma(\{\rho(\bar{n})\}, X)$ are trivial, and have trivial subgame perfect equilibrium utilities. Suppose we have shown, for some $k=2, \ldots, \bar{n}$, that all sequential share bargaining games $\Gamma\left(\rho^{k}, X\right)$ have unique subgame perfect equilibrium utilities.

Consider a game $\Gamma(N, X)$, where $N=\rho^{k-1}$. For $x \in[0, X]$, we replace all subgames $\Gamma\left(\rho^{k}, X-x\right)$ of the game $\Gamma(N, X)$ by their subgame perfect equilibrium utilities. The resulting reduced game is denoted $\hat{\Gamma}(N, X)$. As we show in the proof of Theorem 6.1 , the game thus obtained is a one-dimensional bargaining game with coalitions $S=\{\rho(k-1)\}$ and $T=\rho^{k}$. Subgame perfect equilibrium utilities of $\Gamma(N, X)$ are therefore unique by Theorem 4.1, and a subgame perfect equilibrium of $\Gamma(N, X)$ is found using the characteristic equations for the one-dimensional bargaining game.

A subgame perfect equilibrium strategy profile $\sigma^{*}$ of $\Gamma(\bar{N}, \bar{X})$ is defined as follows. Suppose the set of active players is $N$ and the cake size is $X$, where $N=\rho^{k}$ for some $k=1, \ldots, \bar{n}$, and $0 \leq X \leq \bar{X}$. Let $S=\{\rho(k)\}$ and $T=\rho^{k+1}$. Let $z_{C}^{i}(N, X)$ for $i \in N$ and $C \in\{S, T\}$ denote the solution to the system (1)-(2) of characteristic equations with the matrix of transition probabilities equal to $\pi^{k}$. Then player $\rho(k)$ makes a proposal $x_{i}=X-\delta z_{T}^{i}(N, X)$ and accepts a proposal $x_{j}$ of player $j \in T$ if and only if $x_{j} \geq \delta z_{S}^{j}(N, X)$. Player $i$ of coalition $T$ makes a proposal $x_{i}=\delta z_{S}^{i}(N, X)$ and accepts a proposal $x_{j}$ of player $j \in N$ if and only if $x_{j} \leq X-\delta z_{T}^{j}(N, X)$. In particular, equilibrium proposals are unanimously accepted.

Theorem 6.1 The strategy profile $\sigma^{*}$ is a subgame perfect equilibrium of the game $\Gamma$. The subgame perfect equilibrium utilities of $\Gamma$ are unique. In any subgame perfect equilibrium, agreement is reached without delay.

Proof. We show first that the subgame perfect equilibrium utilities of $\Gamma$ are uniquely determined. The proof of Theorem 6.1 is by induction on the number of active players in the subgames. It is trivially true that for all one-player subgames $\Gamma\left(\rho^{\bar{n}}, X\right)$ discounted equilibrium utilities are unique, and are linear as a function of $X$. Suppose this statement is true for all games with set of players $\rho^{k}$, where $2 \leq k \leq \bar{n}$. Let $u_{i}\left(\rho^{k}, X\right)$ denote the discounted equilibrium utility of player $i \in \rho^{k}$ in the game $\Gamma\left(\rho^{k}, X\right)$.

Consider the game $\Gamma\left(\rho^{k-1}, X\right)$. Suppose that in period $t$ the players in $\rho^{k-1}$ unanimously agree to allocate the share $x$ of the cake to player $\rho(k-1)$. In this case player $\rho(k-1)$

obtains $x$ and the remaining players enter the game $\Gamma\left(\rho^{k}, X-x\right)$ in period $t$. Replacing the 
subgame $\Gamma\left(\rho^{k}, X-x\right)$ of the game $\Gamma\left(\rho^{k-1}, X\right)$ by its subgame perfect equilibrium utilities $\delta^{t} x$ for player $\rho(k-1)$ and $\delta^{t} u_{i}\left(\rho^{k}, X-x\right)$ for each $i \in \rho^{k}$, yields a reduced game denoted by $\hat{\Gamma}\left(\rho^{k-1}, X\right)$. The game $\hat{\Gamma}\left(\rho^{k-1}, X\right)$ is a one-dimensional bargaining game as defined in Section 2, with coalitions $S=\{\rho(k-1)\}$ and $T=\rho^{k}$. Because $u_{i}\left(\rho^{k}, \cdot\right)$ is linear, the utility functions $\delta^{t} u_{i}\left(\rho^{k}, X-x\right)$ are for all $i \in \rho^{k}$ equivalent to the utility function $\delta^{t}(X-x)$, so Theorems 4.1 and 4.2 apply. This completes the induction hypothesis and shows that the game $\Gamma$ has uniquely determined subgame perfect equilibrium utilities.

A similar induction argument together with Theorem 3.2 can be used to show that $\sigma^{*}$ is a subgame perfect equilibrium of the game $\Gamma$. This strategy determines the equilibrium utility levels. Since delay of agreement would lead to different utility levels, it follows that in any subgame perfect equilibrium, agreement is reached without delay.

In general, $\Gamma$ may have more than one subgame perfect equilibrium. Since each subgame of $\Gamma$ can be reduced to a one-dimensional bargaining game, the multiplicity is inessential in exactly the same way as for one-dimensional bargaining games. When the set of active players is $\rho^{k}$ and a proposal is made which is unacceptable to some of the players in $\rho^{k}$, i.e. a player $j \in \rho^{k}$ makes a proposal smaller than $\delta z_{\{\rho(k)\}}^{j}\left(\rho^{k}, X\right)$ or a proposal greater than $X-\delta z_{\rho^{k+1}}^{j}\left(\rho^{k}, X\right)$, it does not matter which player in $\rho^{k}$ rejects the proposal. Aggregate behavior is uniquely determined, except in subgames with at least three active players, where by mistake a player $j \in \rho^{k+1}$ proposes $x_{j}=X-\delta z_{\rho^{k+1}}^{j}\left(\rho^{k}, X\right)$, so leaving players in his own coalition indifferent between accepting and rejecting. The other case where aggregate behavior was not uniquely determined in one-dimensional bargaining, where by mistake player $\rho(k)$ proposes $x_{\rho(k)}=\delta z_{\{\rho(k)\}}^{\rho(k)}\left(\rho^{k}, X\right)$, does not occur in sequential share bargaining, since player $\rho(k)$ does not have any coalition members. Such a proposal would be accepted in all subgame perfect equilibria by the members of $\rho^{k+1}$.

We make a final remark with regard to the solution $z_{C}^{i}(N, X)$ to the system (1)-(2) of characteristic equations. Recall that $z_{T}^{i}(N, X)$ is the continuation utility of any player $j$ in $T$ in the game $\hat{\Gamma}(N, X)$ after a proposal by player $i$ has been rejected, relative to the specific utility representation $\delta^{t}(X-x)$ of that player's preferences. To obtain the continuation utility in the game $\Gamma(N, X)$ we must transform $z_{T}^{i}(N, X)$ using the utility function $u_{j}(T, \cdot)$, where $u_{j}(T, X)$ denotes the equilibrium utility of player $j \in T$ in the game $\Gamma(T, X)$. Thus $u_{j}\left(T, z_{T}^{i}(N, X)\right)$ is the continuation utility of player $j \in T$ in the subgame $\Gamma(N, X)$ after a proposal by player $i$ has been rejected. For the unique player in $S$, no transformation is required: $z_{S}^{i}(N, X)$ is the continuation utility of player $S$ after a rejection of a proposal by player $i$.

Example 6.1. Consider a game $\Gamma(\bar{N}, \bar{X})$ where $\bar{N}=\{1, \ldots, \bar{n}\}$. Let $\rho$ be the identity, 
so the sequence in which the players' shares are determined is $1, \ldots, \bar{n}$. In each subgame $\Gamma(N, X)$ with $N=\rho^{k}$ for some $k=1, \ldots, \bar{n}$, the identity of the proposer cycles clockwise within the player set: $k, k+1, \ldots, \bar{n}, k, \ldots$ Thus, when player $k$ is on top of the agenda, it is player $k$ who is the first player to make a proposal. We know that all equilibrium proposals are immediately accepted. This means that the equilibrium proposal $x_{k}$ of player $k$, when player $k$ is on top of the agenda, is also player $k$ 's equilibrium utility.

We claim that

$$
x_{k}=\delta^{k-1} \frac{1-\delta}{1-\delta^{\bar{n}}} \bar{X} .
$$

The argument is by induction on $k$. To see that the claim is true for $k=1$, observe that the reduced game $\hat{\Gamma}(\bar{N}, \bar{X})$ is the one-dimensional bargaining game of Example 3.1 with $S=\{1\}, T=\{2, \ldots, \bar{n}\}$, and cake size $\bar{X}$. The formula for $x_{1}$ then follows from the result of Example 3.1. Suppose the claim is true for all $i=1, \ldots, k$. We prove that it holds for $i=k+1$.

Suppose that the players $1, \ldots, k$ have left the game. The remaining players $\rho^{k+1}=$ $\{k+1, \ldots, \bar{n}\}$ are then dividing a cake of size $X=\bar{X}-x_{1}-\cdots-x_{k}$. Using the induction hypothesis, this can be seen to be equal to

$$
X=\delta^{k} \frac{1-\delta^{\bar{n}-k}}{1-\delta^{\bar{n}}} \bar{X}
$$

The reduced game $\hat{\Gamma}(N, X)$ is the one-dimensional bargaining game of Example 3.1 with $S=\{k+1\}$ and $T=\{k+2, \ldots, \bar{n}\}$. Player $k+1$ then makes a proposal

$$
x_{k+1}=\frac{1-\delta}{1-\delta^{\bar{n}-k}} X=\delta^{k} \frac{1-\delta}{1-\delta^{\bar{n}}} \bar{X},
$$

which establishes the claim.

Example 6.2. Consider a game $\Gamma(\bar{N}, \bar{X})$ where $\bar{N}=\{1, \ldots, \bar{n}\}$. As in the previous example, the agenda $\rho$ equals the identity, so the sequence in which the players' shares are determined is $1, \ldots, \bar{n}$. The proposer is chosen randomly from the set of active players with equal probabilities.

We claim that the expected equilibrium utility of any player is $\bar{X} / \bar{n}$. First we show that the expected equilibrium utility of player 1 is $\bar{X} / \bar{n}$. The reduced game $\hat{\Gamma}(\bar{N}, \bar{X})$ is the one-dimensional bargaining game of Example 3.2 with $S=\{1\}, T=\{2, \ldots, \bar{n}\}$, and cake size $\bar{X}$. We know that in the game $\hat{\Gamma}(\bar{N}, \bar{X})$ the equilibrium proposal of player 1 is $[\bar{n}-\delta(\bar{n}-1)] \bar{X} / \bar{n}$, while the equilibrium proposal of any member of coalition $T$ is $\delta \bar{X} / \bar{n}$. Since the identity of the proposer is uniformly distributed in $\bar{N}$, the expected share of player 1 , which is also player 1's expected utility, is

$$
\frac{1}{\bar{n}} \frac{\bar{n}-\delta(\bar{n}-1)}{\bar{n}} \bar{X}+\frac{(\bar{n}-1)}{\bar{n}} \frac{\delta}{\bar{n}} \bar{X}=\frac{\bar{X}}{\bar{n}} \text {. }
$$


Suppose the claim is true for all $i=1, \ldots, k$. We prove that it holds for $i=k+1$. Suppose the players $1, \ldots, k$ have left the game with shares $x_{1}, \ldots, x_{k}$. The remaining players $N=\{k+1, \ldots, \bar{n}\}$ are then dividing a cake of size $X=\bar{X}-x_{1}-\cdots-x_{k}$. Now the shares $x_{1}, \ldots, x_{k}$ and the leftover $X$ are random functions, as they depend on the moves of nature (the $k$ choices of proposers prior to the exit of player $k$ ). Because by the induction hypothesis the expected value of $x_{i}$ for each $i=1, \ldots, k$ is $\bar{X} / \bar{n}$, the expected value of $X$ is $(\bar{n}-k) \bar{X} / \bar{n}$.

Given a specific realization of $X$, consider a reduced game $\hat{\Gamma}(N, X)$. This is the onedimensional bargaining game of Example 3.2 with $S=\{k+1\}$ and $T=\{k+2, \ldots, \bar{n}\}$. Notice that the cardinality of $N$ is $\bar{n}-k$. We know that in $\hat{\Gamma}(N, X)$ the equilibrium proposal of player $k+1$ is $(\bar{n}-k-\delta(\bar{n}-k-1)) X /(\bar{n}-k)$ and the equilibrium proposal of each member of coalition $T$ is $\delta X /(\bar{n}-k)$. Since the identity of the proposer is uniformly distributed on $N$, the expected share of player $k$ conditional on $X$ is

$$
\frac{1}{\bar{n}-k} \frac{\bar{n}-k-\delta(\bar{n}-k-1)}{\bar{n}-k} \bar{X}+\frac{(\bar{n}-k-1)}{\bar{n}-k} \frac{\delta}{\bar{n}-k} X=\frac{X}{\bar{n}-k} .
$$

This expression is linear in $X$. To compute the unconditional expected share (or utility) of player $k$, we substitute the expected value of $X$ for $X$ in the formula above. This yields $\bar{X} / \bar{n}$, as desired.

\section{Conclusions}

The existing results on $n$-player bargaining problems with unanimous agreement point towards a large multiplicity of subgame perfect equilibria. This is a very unpleasant result, since it implies that the predictive power of the model is extremely limited.

There are several ways to extend the Rubinstein-Ståhl bargaining model for 2 players to the case with $n$ players. This paper considers the case where the shares of the players are not determined simultaneously, but sequentially, thereby removing a potential source of multiplicity of equilibria.

The paper obtains unique subgame perfect equilibrium utilities for this bargaining procedure. In equilibrium, proposals are accepted without delay. Our results for $n$ players are qualitatively the same as the results for the two player case. The paper also studies a related class of bargaining problems, called one-dimensional bargaining problems, and obtains a uniqueness result there as well.

Our results imply that the choice of the bargaining procedure is important in obtaining desirable bargaining outcomes. The idea of determining the players' shares sequentially is 
natural, and avoids the coordination problem that occurs when all shares are determined at the same time.

\section{References}

Banks, J., and J. Duggan (2000), "A Bargaining Model of Collective Choice," American Political Science Review, 94, 73-88.

Chae, S., and J.-A. Yang (1988), "The Unique Perfect Equilibrium of an N-Person Bargaining Game," Economics Letters, 28, 221-223.

Chae, S., and J.-A. Yang (1994), "An N-Person Pure Bargaining Game," Journal of Economic Theory, 62, 86-102.

Cho, S.J., and J. Duggan (2003), "Uniqueness of Stationary Equilibria in a One-dimensional Model of Bargaining," Journal of Economic Theory, 113, 118-130.

Haller, H. (1986), Non-cooperative Bargaining of $N \geq 3$ Players, Economics Letters, 22, $11-13$.

Herrero, M. (1985), A Strategic Bargaining Approach to Market Institutions, PhD Thesis, London School of Economics, London.

Huang, C.-Y. (2002), "Multilateral Bargaining: Conditional and Unconditional Offers," Economic Theory, 20, 401-412.

Jun, B.H. (1987), A Strategic Model of 3-Person Bargaining, Unpublished Paper, State University of New York at Stony Brook.

Krishna, V., and R. Serrano (1996), "Multilateral Bargaining," Review of Economic Studies, 63, 61-80.

Merlo, A., and C. Wilson (1995), "A Stochastic Model of Sequential Bargaining with Complete Information," Econometrica, 63, 371-399.

Osborne, M.J., and A. Rubinstein (1990), Bargaining and Markets, Academic Press, San Diego.

Rubinstein, A. (1982), "Perfect Equilibrium in a Bargaining Model," Econometrica, 50, 97-109.

Ståhl, I. (1972), Bargaining Theory, Economics Research Institute, Stockholm School of 
Economics, Stockholm.

Suh, S.-C., and Q. Wen (2006), "Multi-agent Bilateral Bargaining and the Nash Bargaining Solution," Journal of Mathematical Economics, 42, 61-73.

Yang, J.-A. (1992), "Another $n$-Person Bargaining Game with a Unique Perfect Equilibrium," Economics Letters, 38, 275-277. 